УДК 616.12-007.61

DOI: $10.15587 / 2313-8416.2015 .36015$

\title{
СОСТОЯНИЕ ОРГАНОВ-МИШЕНЕЙ ПРИ РАЗЛИЧНЫХ ФОРМАХ АРТЕРИАЛЬНОЙ ГИПЕРТЕНЗИИ У БЕРЕМЕННЫХ
}

\author{
(C) В. С. Чулков
}

Проведена оценка состояния органов-мишеней у 95 беременных с различными формами артериальной гипертензии. Показано, что наиболее ранним и потенциально обратимым поражением органов-мишеней при артериальной гипертензии у беременных независимо от ее формы является гипертрофия левого желудочка. Обнаружение микроальбуминурии в зависимости от сроков беременности может служить дополнительным критерием в пользу преэклампсии

Ключевые слова: артериальная гипертензия, беременность, гипертрофия левого желудочка, микроальбуминурия, толщина комплекса интима-медиа

We have estimated the state of target-organ damage in 95 pregnant women with different forms of hypertension. The early and potentially reversible pathophysiological and pathomorphological changes of the target organs in pregnant women with hypertension are structural and functional changes of the heart and kidneys. To assess the state of the target organs we were examined the blood vessels of the ocular fundus, the presence of left ventricular hypertrophy, the thickness of the intima-media complex and the presence of microalbuminuria. It was shown that the earliest target-organ damage in pregnant women with hypertension was left ventricular hypertrophy regardless of its form. Detection of microalbuminuria depending on gestational age may serve as an additional criterion of pre-eclampsia. With consideration of the high frequency changes in the retinal vessels, especially in preeclampsia superimposed on chronic hypertension, is required further researches to evaluate the prognostic significance of these changes. Assessment of target organs in pregnant women with hypertension can be used as a surrogate prediction point of complications and adverse pregnancy outcomes

Keywords: arterial hypertension, pregnancy, left ventricular hypertrophy, microalbuminuria, the thickness of the intima-media complex

\section{1. Введение}

Гипертензивные нарушения остаются главной причиной материнской и младенческой заболеваемости и смертности в развитых и развивающихся странах $[1,2]$. Женщины с артериальной гипертензией (АГ) имеют более высокий риск развития таких тяжелых осложнений, как острые нарушения мозгового кровообращения, сердечно-сосудистые осложнения, почечная недостаточность, диссеминированное внутрисосудистое свертывание крови и ряд прочих [3, 4]. Наиболее ранними и потенциально обратимыми патофизиологическими и патоморфологическими изменениями со стороны органов-мишеней при АГ у беременных являются структурно-функциональные изменения сердца и почек [5-7].

\section{2. Постановка проблемы}

В последние годы важными маркерами, определяющими прогноз у пациентов АГ, признаны такие признаки субклинического поражения органов, как гипертрофия миокарда левого желудочка (ГЛЖ), микроальбуминурия (МАУ) и ультразвуковые признаки поражения сонных артерий в виде увеличения комплекса интима/медиа $[8,9]$. В современной литературе имеются немногочисленные исследования, отражающие оценку состояния органов-мишеней при различных формах АГ у беременных $[5,6,10]$.

Цель исследования - провести оценку состояния органов-мишеней у беременных с различными формами артериальной гипертензии.

\section{3. Литературный обзор}

Комплекс структурно-функциональных изменений со стороны сердца при АГ в настоящее время объединяют под термином ремоделирование, в понятие которого входят изменения размеров, формы, структуры, а также биохимических и функциональных свойств миокарда левого желудочка [9, 11-13]. Другим важным органом-мишенью при АГ являются почки. Микроальбуминурия является наиболее ранним маркером поражения почек при АГ, а также предиктором неблагоприятного течения заболевания и фактором высокого риска развития сердечнососудистых осложнений в последующем [8, 14]. Установление факта наличия МАУ позволяет выделять прогностически наиболее тяжелую группу пациентов, которая требует пристального наблюдения и адекватного лечения [15]. Однако, если в доступной литературе оценке состояния сердца уделено важное значение, то определению МАУ у беременных придается пока намного меньшая роль, несмотря на высокую диагностическую значимость.

\section{4. Материалы и методы}

Тип исследования: случай-контроль.

Источниковой популяцией явились беременные, наблюдавшиеся в городском отделе патологии гемостаза на базе МБУЗ «Городская клиническая больницы № 11» г. Челябинска за период 2009-2012 гг.

Метод выборки: сплошной по мере обращаемости. 
Критерии включения: беременность на момент включения до 20 недель; возраст от 18 до 45 лет; наличие артериальной гипертензии; информированное согласие пациентки на участие в исследовании.

Критерии исключения: симптоматические артериальные гипертензии; тяжелые соматические заболевания; системные заболевания соединительной ткани; ментальные расстройства и психические заболевания; хронический алкоголизм, наркомания.

Артериальная гипертензия устанавливалась на основании повышения систолического артериального давления (САД) $\geq 140$ мм рт. ст. и/или диасто-лического артериального давления (ДАД) $\geq 90$ мм рт. ст., зарегистрированных при двух и более последовательных измерениях с интервалом $\geq 4$ часов $[2,16]$.

В соответствии с критериями включения/исключения в исследование вошли 95 беременных с АГ, которые были разделены на 4 группы: группа 1-45 женщин с хронической АГ (ХАГ), группа 2-20 женщин с гестационной АГ (ГАГ), группа 310 женщин с преэклампсией, группа 4-15 женщин с преэклампсией на фоне ХАГ. В группу контроля вошли 35 женщин без АГ, не имевших отягощенного акушерско-гинекологического анамнеза.

Проводились сбор анамнеза, клиническое обследование, анализ медицинской документации, стандартное лабораторно-инструментальное обследование. Для диагностики поражений органовмишеней проводились: осмотр сосудов глазного дна, стандартная ЭКГ в 12-ти отведениях, эхокардиография (ультразвуковой аппарат «Sonos 100», «НР», Германия), ультразвуковое исследование сонных артерий (ультразвуковой сканер «My Lab 20», «Esaote», Италия), общий анализ мочи для диагностики протеинурии, анализ мочи на МАУ, анализ крови на креатинин. Выявление МАУ производили иммунологическим полуколичественным методом при помощи тест-полосок Micral-test («Roche Diagnostics GmbH», Германия). Положительным считался результат на МАУ>20 мг/л, определенный дважды. Гипертрофия левого желудочка (ГЛЖ) диагностировалась по ЭКГ или ультразвуковым критериям: признак СоколоваЛайона более 38 мм, Корнельское произведение более 2440 мм $\times$ мс; ИММ ЛЖ $\geq 110$ г/м² (по R. Devereux). Ультразвуковое исследование сонных артерий выполнялось в положении больного лежа с линейным датчиком 7 мГц при незначительном отклонении головы пациента в противоположную сторону. Измерения проводились в В-режиме в продольном сечении по дальней стенке артерии на расстоянии от 1,0 до 1,5 см проксимальнее бифуркации общей сонной артерии (ОСА). За норму принимали показатель толщины комплекса интима-медиа (ТИМ) ОСА менее 0,9 мм.

Статистический анализ данных проводился при помощи пакета статистических программ MedCalc® версия 11.5 .0 (2011). Данные в тексте представлены в виде средней арифметической и ее среднеквадратичного отклонения $(\mathrm{M} \pm \sigma)$. Применялись критерии Стьюдента, Краскелла-Уоллиса, критерий $\chi^{2}$ (хи- квадрат) в зависимости от типа данных и характера распределения. Для всех видов анализа статистически достоверными считались значения $\mathrm{p}<0,05$.

\section{5. Апробация результатов исследований}

Возраст пациенток составил $31,5 \pm 5,0$ лет у женщин с ХАГ, 25 $\pm 3,9$ лет - у женщин с ГАГ, $25 \pm 3,6$ лет - у женщин с преэклампсией, $31 \pm 5,3$ лет у

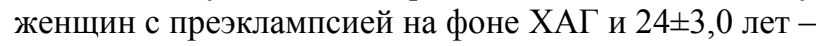
в контрольной группе $\left(\mathrm{p}_{1,4-2,3, \mathrm{\kappa}}<0,001\right)$.

Частота встречаемости факторов сердечнососудистого риска у беременных с различными формами АГ представлена в табл. 1.

Самым распространенным фактором риска оказалась отягощенная наследственность по АГ, особенно у беременных с хронической АГ (группы 1 и 4). Далее по частоте встречаемости отмечались ожирение и избыточная масса тела, а также курение до беременности во всех группах АГ. Отягощенная наследственность по ранним сердечно-сосудистым заболеваниям чаще отмечалась в группах с хронической АГ (группы 1 и 4) по сравнению с контрольной группой.

Выявленные при обследовании поражения органов-мишеней представлены в табл. 2.

Наиболее часто в исследуемых группах было выявлено поражение сосудов глазного дна в виде сужения артериол сетчатки, особенно у беременных с $\mathrm{XАГ} \mathrm{с} \mathrm{присоединившейся} \mathrm{преэклампсией.} \mathrm{В} \mathrm{совре-}$ менных рекомендациях в верификацию стадии АГ поражение сосудов сетчатки не входит, однако, во многих рекомендациях по диагностике и лечению АГ орган зрения однозначно трактовался как органмишень в связи со специфичностью офтальмоскопических изменений, имеющих прогностическое значение. Существующая несогласованность требует продолжения исследований в этой области.

Гипертрофия левого желудочка примерно одинаково встречалась в группах со всеми формами АГ по сравнению с контролем. Во время беременности наличие ГЛЖ или ее формирование за короткий период, ограниченный гестационным периодом, могут формировать предпосылки для развития преэклампсии.

Микроальбуминурия, с одной стороны, является проявлением поражения почек при АГ, с другой стороны - расценивается как проявление системной эндотелиальной дисфункции $[5,6,14]$. Во время беременности определение МАУ также имеет неоднозначную трактовку на разных сроках гестации: выявление МАУ до 20 недель у беременных впервые может отражать поражение почек, как органамишени при АГ, а обнаружение МАУ только на сроках после 20 недель - предиктором преэклампсии. В нашем исследовании у беременных с ХАГ МАУ выявлялась у 13,3 \% женщин на сроках до 20 недель при отсутствии ее нарастания в III триместре беременности, что отражает поражение почек при АГ, а у женщин с преэклампсией и преэклампсией на фоне ХАГ - впервые только после 20 недель. Это позволяет говорить о МАУ, как раннем предикторе развития преэклампсии. 
Таблица 1

Частота встречаемости факторов сердечно-сосудистого риска у беременных с различными формами артериальной гипертензии

\begin{tabular}{|c|c|c|c|c|c|c|c|c|c|c|}
\hline \multirow[t]{2}{*}{ Показатель } & \multicolumn{2}{|c|}{$\begin{array}{c}\text { Группа } 1 \\
(\mathrm{n}=45)\end{array}$} & \multicolumn{2}{|c|}{$\begin{array}{c}\text { Группа } 2 \\
(\mathrm{n}=20)\end{array}$} & \multicolumn{2}{|c|}{$\begin{array}{c}\text { Группа } 3 \\
(\mathrm{n}=10)\end{array}$} & \multicolumn{2}{|c|}{$\begin{array}{c}\text { Группа } 4 \\
(\mathrm{n}=15)\end{array}$} & \multicolumn{2}{|c|}{$\begin{array}{c}\text { Контроль } \\
(\mathrm{n}=35)\end{array}$} \\
\hline & $\mathrm{N}$ & $\%$ & $\mathrm{~N}$ & $\%$ & $\mathrm{~N}$ & $\%$ & $\mathrm{~N}$ & $\%$ & $\mathrm{~N}$ & $\%$ \\
\hline Избыточ & 34 & $75,5^{*}$ & 14 & $70,0^{*}$ & 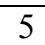 & $50,0^{*}$ & 11 & 73,0 * & 1 & 2,3 \\
\hline Курение до беременности & 13 & $28,9^{*}$ & 3 & $15,0^{*}$ & 2 & $20,0^{*}$ & 4 & $26,7 *$ & 0 & 0 \\
\hline Отягощенная наследственность по АГ & 33 & $73,3^{*}$ & 5 & 25,0 & 6 & $60,0^{*}$ & 14 & $93,3^{*}$ & 3 & 8,6 \\
\hline $\begin{array}{l}\text { Отягощенная наследственность по } \\
\text { ранним сердечно-сосудистым заболе- } \\
\text { ваниям }\end{array}$ & 9 & $20,0^{*}$ & 2 & 10,0 & 2 & 20,0 & 7 & $46,7^{*}$ & 1 & 2,9 \\
\hline
\end{tabular}

Примечание: *-p<0,05 - при сравнении с контрольной группой

Таблица 2

Частота обнаружения поражения органов мишеней у беременных в группах сравнения

\begin{tabular}{|c|c|c|c|c|c|c|c|c|c|c|}
\hline \multirow[t]{2}{*}{ Показатель } & \multicolumn{2}{|c|}{$\begin{array}{c}\text { Группа } 1 \\
(\mathrm{n}=45)\end{array}$} & \multicolumn{2}{|c|}{$\begin{array}{c}\text { Группа } 2 \\
(\mathrm{n}=20)\end{array}$} & \multicolumn{2}{|c|}{$\begin{array}{c}\text { Группа } 3 \\
(\mathrm{n}=10)\end{array}$} & \multicolumn{2}{|c|}{$\begin{array}{c}\text { Группа } 4 \\
(\mathrm{n}=15)\end{array}$} & \multicolumn{2}{|c|}{$\begin{array}{c}\text { Контроль } \\
(\mathrm{n}=35)\end{array}$} \\
\hline & $\mathrm{N}$ & $\%$ & $\mathrm{~N}$ & $\%$ & $\mathrm{~N}$ & $\%$ & $\mathrm{~N}$ & $\%$ & $\mathrm{~N}$ & $\%$ \\
\hline Сужение артериол сетчатки & 26 & $57,7 *$ & 10 & $50,0^{*}$ & 4 & $40,0^{*}$ & 14 & $93,3^{*}$ & 2 & 2,0 \\
\hline $\begin{array}{l}\text { Гипертрофия левого желу- } \\
\text { дочка }\end{array}$ & 6 & $13,3^{*}$ & 3 & $15,0^{*}$ & 2 & $20,0^{*}$ & 2 & 13,3 & 0 & 0 \\
\hline Поражение сонных артерий & 3 & 8,7 & 0 & 0 & 1 & 10,0 & 2 & 13,3 & 0 & 0 \\
\hline Микроальбуминурия & 6 & $13,3^{*}$ & 0 & 0 & 3 & $30,0^{*}$ & 6 & $40,0^{*}$ & 0 & 0 \\
\hline
\end{tabular}

Примечание: * $-p<0,05-$ при сравнении с контрольной группой

Реже всего встречалось поражение сонных артерий (увеличение комплекса интима-медиа более 0,9 мм) в группах с ХАГ, преэклампсией и преэклампсией на фоне ХАГ, что, с одной стороны, может быть объяснено относительно молодым возрастом исследуемых женщин, с другой стороны - небольшой длительностью АГ (стажем АГ).

В контроле изменений, отражающих поражение органов-мишеней и входящих в стратификацию стадии АГ, не обнаружено.

\section{6. Выводы}

1. Наиболее ранним поражением органовмишеней при артериальной гипертензии у беременных оказалась гипертрофия левого желудочка. В нашем исследовании гипертрофия левого желудочка (по данным эхокардиографии) встречалась у 13,7 \% беременных с артериальной гипертензией, имея примерно одинаковую частоту встречаемости при различных формах артериальной гипертензии.

2. Наиболее частым поражением органовмишеней, особенно при хронической артериальной гипертензии с присоединением преэклампсии, явилось поражение органа зрения в виде сужения артериол сетчатки. Ввиду отсутствия данного критерия в верификации стадии артериальной гипертензии в общей популяции, требуются дальнейшие исследования по оценке прогностической значимости этого поражения органа-мишени у беременных с артериальной гипертензией.

3. Обнаружение микроальбуминурии может использоваться для дифференциальной диагностики различных форм артериальной гипертензии: при ее обнаружении до 20 недель можно говорить о поражении почек при II или III стадии артериальной гипертензии, а выявление впервые после 20 недель может быть ранним критерием преэклампсии.

4. Оценка состояния сонных артерий по измерению толщины комплекса интима-медиа у беременных реже всего выявляет изменения, соответствующие II стадии артериальной гипертензии, однако она необходима в комплексной изучении состояния органов-мишеней.

\section{Литература}

1. Долгушина, В. Ф. Акушерские осложнения при различных формах артериальной гипертензии у беременных [Текст] / В. Ф. Долгушина, В. С. Чулков, Н. К. Вереина, С. П. Синицын // Акушерство и гинекология. - 2013. № 10. - C. 33-39.

2. Magee, L. A. Diagnosis, evaluation, and management of the hypertensive disorders of pregnancy [Text] / L. A. Magee, A. Pels, M. Helewa, E. Rey, P. von Dadelszen // Pregnancy Hypertension: An International Journal of Women's Cardiovascular Health. - 2014. - Vol. 4, Issue 2. - P. 105-145. doi: 10.1016/j.preghy.2014.01.003

3. Чулков, В. С. Особенности течения и исходов беременности у женщин с различными формами артериальной гипертензии [Текст] / В. С. Чулков // Аспирантский вестник Поволжья. - 2009. - № 7-8. - С. 75-79.

4. Чулков, В. С. Особенности структуры, анамнеза и исходы беременности при артериальной гипертензии [Текст] / В. С. Чулков, С. П. Синицын, Н. К. Вереина, Н. В. Уразова // Экология человека. - 2009. - № 10. -. - С. 49-54.

5. Ткачева, О. Н. Актуальные вопросы патогенеза, диагностики и фармакотерапии артериальной гипертонии у беременных [Текст] / О. Н. Ткачева, А. В. Барабашкина. М.: Пагри, 2006. - 140 c. 
6. Синицын, С. П. Артериальная гипертензия у беременных с факторами тромбогенного риска: особенности течения различных клинических форм, состояния органовмишеней и показателей гемостаза [Текст] / С. П. Синицын, В. С. Чулков, Н. К. Вереина // Артериальная гипертензия. 2009. - T. 15, № 5. - С. 580-584.

7. Чулков, В. С. Изучение показателей внутрисердечной гемодинамики и лабораторных маркеров эндотелиальной дисфункции у беременных с артериальной гипертензией [Текст] / В. С. Чулков, С. П. Синицын, Н. К. Вереина, Д. Н. Гафурова // Уральский медицинский журнал. - 2008. - № 5 (45). - С. 26-30.

8. Ochodnicky, P. Microalbuminuria and endothelial dysfunction: emerging targets for primary prevention of end-organ damage [Text] / P. Ochodnicky, R. H. Henning, R. P. van Dokkum et al. // Journal of Cardiovascular Pharmacology. - 2006. - Vol. 47, Suppl. 2. - P. S151-S162. doi: 10.1097/00005344-20060600100009

9. Ganau, A. Patterns of left ventricular hypertrophy and geometric remodeling in essential hypertension [Text] / A. Ga-nau, R. V. Devereux, M. J. Roman // Journal of the American College of Cardiology. - 1992. - Vol. 19, Issue 7. - P. 1550-1558. doi: 10.1016/0735-1097(92)90617-v

10. Schannwell, C. M. Left ventricular hypertrophy and diastolic dysfunction in healthy pregnant women [Text] / C. M. Schannwell, T. Zimmermann, M. Schneppenheim et al. // Cardiology. - 2002. - Vol. 97, Issue 2. - P. 73-78. doi: $10.1159 / 000057675$

11. Вебер, В. Р. Диастолическая дисфункция левого и правого желудочка у больных с артериальной гипертонией и возможности их коррекции [Текст] / В. Р. Вебер, М. П. Рубанова, С. В. Жмайлова и др. // Сердечная недостаточность. -2005 . - № 3. - С. 107-109.

12. Maisch, B. Ventricular remodeling [Text] / B. Maisch // Cardiology. - 1996. - Vol. 87, Issue 1. - P. 2-10. doi: $10.1159 / 000177160$

13. Гипертоническое сердце [Текст] / под ред акад. Р. Г. Оганова, проф. Э. Г. Волковой. - М.: ООО «Компания БОРГЕС», 2008. - $212 \mathrm{c}$.

14. Taddei, S. Lack or correlation between microalbuminuria and endothelial function in essential hypertensive patients [Text] / S. Taddei, A. Virdis, P. Mattei et al. // Journal of Hypertension. - 1995. - Vol. 13, Issue 9. - P. 1003-1008. doi: 10.1097/00004872-199509000-00010

15. Bar, J. Microalbuminuria after pregnancy complicated by preeclampsia [Text] / J. Bar, B. Kaplan, C. Wittenberg // Nephrology Dialysis Transplantation. - 1999. - Vol. 14, Issue 5. P. 1129-1132. doi: 10.1093/ndt/14.5.1129

16. Диагностика и лечение сердечно-сосудистых заболеваний при беременности. Российские рекомендации [Текст] / Российский кардиологический журнал. - 2013. - № 4 (102), приложение 1. - С. 1-40.

\section{References}

1. Dolgushina, V. F., Chulkov, V. S., Vereina, N. K., Sinitsin, S. P. (2013). Obstetric complications in different forms of hypertension in pregnancy. Obstetrics and Gynecology, 10, 33-39.
2. Magee, L. A., Pels, A., Helewa, M., Rey, E., von Dadelszen, P. (2014). Diagnosis, evaluation, and management of the hypertensive disorders of pregnancy. Pregnancy Hypertension: An International Journal of Women's Cardiovascular Health. 2014. - Vol. 4, Issue 2. - P. 105-145. doi: 10.1016/j.preghy.2014.01.003

3. Chulkov, V. S. (2009). Peculiarities of the course and outcomes of pregnancy in women with different forms of hypertension. Graduate Bulletin of the Volga region, 7-8, 75-79.

4. Chulkov, V. S., Sinitsin, S. P., Vereina, N. K. (2009). Features of the structure, history and pregnancy outcomes in hypertension. Human Ecology, 10, 49-54.

5. Tkacheva, O. N., Barabashkina, A. V. (2006). Current issues of pathogenesis, diagnosis and pharmacotherapy of hypertension in pregnancy. Moscow: Pagri, 140.

6. Sinitsyn, S. P. Chulkov, V. S., Vereina, N. K. (2009). Hypertension in women with thrombogenic factors: the peculiarities of different forms, condition of target organs and hemostatic parameters. Arterial hypertension, 15 (5), 580-584.

7. Chulkov, V. S., Sinitsyn, S. P., Vereina, N. K., Gafurova, D. N. (2009). A study of intracardiac hemodynamics parameters and laboratory markers of endothelial dysfunction in pregnant women with arterial. Ural medical journal, 5 (45), 26-30.

8. Ochodnicky, P., Henning, R. H., van Dokkum, R. P. et al. (2006). Microalbuminuria and endothelial dysfunction: emerging targets for primary prevention of end-organ damage. Journal of Cardiovascular Pharmacology, 47 (Suppl. 2), S151-S162. doi: 10.1097/00005344-200606001-00009

9. Ganau, A. A., Devereux, R. V., Roman, M. J. (1992). Patterns of left ventricular hypertrophy and geometric remodeling in essential hypertension. Journal of the American College of Cardiology, 19 (7), 1550-1558. doi: 10.1016/0735-1097(92)90617-v

10. Schannwell, C. M., Zimmermann, T., Schneppenheim, M. et al. (2002). Left ventricular hypertrophy and diastolic dysfunction in healthy pregnant women. Cardiology, 97 (2), 73 78. doi: $10.1159 / 000057675$

11. Weber, C. R., Rubanov, M. P., Smailova, S. C. (2005). Diastolic dysfunction of left and right ventricles in patients with arterial hypertension and possibilities of their correction. Heart failure, 3, 107-109.

12. Maisch, B. (1996). Ventricular remodeling. Cardiology, 87 (1), 2-10. doi: 10.1159/000177160

13. Oganov, R. G., Volkova, E. G. (2008). Hypertensive heart. Moscow: Borges, 212.

14. Taddei, S. A., Virdis, A., Mattei, P. et al. (1995). Lack or correlation between microalbuminuria and endothelial function in essential hypertensive patients. Journal of Hypertension, 13, 1003-1008. doi: 10.1097/00004872-199509000-00010

15. Bar, J., Kaplan, B., Wittenberg, C. (1999). Microalbuminuria after pregnancy complicated by preeclampsia. Nephrology Dialysis Transplantation, 14 (5), 1129-1132. doi: $10.1093 / \mathrm{ndt} / 14.5 .1129$

16. Diagnosis and treatment of cardiovascular diseases during pregnancy. Russian recommendations (2013). Russian Journal of Cardiology, 4 (102), application 1, 1-40.

Рекомендовано к публикации д-р мед. наук Синицын С. П. Дата поступления рукописи 25.12.2014

Чулков Василий Сергеевич, кандидат медицинских наук, доцент, кафедра факультетской терапии, ГБОУ ВПО «Южно-Уральский государственный медицинский университет» Минздрава РФ, ул. Воровского, 64, г. Челябинск, Россия, 454092

E-mail: vschulkov@rambler.ru 